\title{
Degradation Analysis and Simulation of Li-ion Batteries
}

\author{
Wei Lu \\ Department of Mechanical Engineering, University of Michigan, Ann Arbor, USA \\ Department of Materials Science \& Engineering, University of Michigan, Ann Arbor, USA \\ weilu@umich.edu
}

The electrification of the drivetrain is crucial to solve our energy problems and fill the gap to sustainable and zero emission mobility. We are now within reach of mass-marketed vehicles using Li-based batteries. Full engineering analysis of batteries and battery systems must be regularized, and engineering development is needed to allow creation of products and tools for integration of battery systems into vehicles. Electric vehicles and hybrid electric vehicles face significant battery-related challenges, including limited driving range and high battery cost resulting from the capacity fade of batteries during usage. The prediction of capacity fade and lifetime of batteries is important for cell design, determination of the optimal operation condition and control, and cell maintenance. Various mechanisms contribute to capacity fade. An integrated approach considering different aspects of the fading mechanisms is necessary. In this talk I will present some of our recent work in these areas, including a comprehensive capacity fade model and its experimental validation and application for battery optimization; a multiscale approach that couples mechanics and electrochemistry consistently at both particle and electrode scales which enables simulating various electrode phenomena, and its validation against explicit simulation of particle networks; and simulation of concurrent dendrite growth, SEI formation and penetration of lithium metal electrodes; and a new materials strategy to stop dendrite formation by a soft piezoelectric film. The materials strategy for improving the battery performance and application of machine learning for battery design will also be discussed. 